\section{The $\mathrm{Si}_{3} \mathrm{~N}_{4} / \mathrm{TiN}$ Interface: 7. Ti/TiN(001) Grown and Analyzed In situ using X-ray Photoelectron Spectroscopy}

\author{
Richard T. Haasch ${ }^{\text {a) }}$ \\ University of Illinois, Department of Materials Science and Frederick Seitz Materials Research \\ Laboratory, 104 S. Goodwin Avenue, Urbana, IL 61801, USA
}

Jörg Patscheider

University of Illinois, Department of Materials Science and Frederick Seitz Materials Research Laboratory, 104 S. Goodwin Avenue, Urbana, IL 61801, USA and EMPA, Laboratory for Nanoscale Materials Science, Überlandstrasse 129 CH-8600, Dübendorf, Switzerland

Niklas Hellgren

University of Illinois, Department of Materials Science and Frederick Seitz Materials Research Laboratory, 104 S. Goodwin Avenue, Urbana, IL 61801, USA and Messiah College, Department of Mathematical Sciences, P.O. Box 3041, One College Avenue, Grantham, PA 17027, USA

Ivan Petrov and J. E. Greene

University of Illinois, Department of Materials Science and Frederick Seitz Materials Research Laboratory, 104 S. Goodwin Avenue, Urbana, IL 61801, USA

(Received 11 October 2012; accepted 21 November 2012; published 19 December 2012)

Accession \#: 01246
Technique: XPS
Host Material: Ti/TiN(001) bilayer
Instrument: Kratos Axis Ultra
Major Elements in Spectra: Ti, N
Minor Elements in Spectra: O, C
Published Spectra: 5
Spectra in Electronic Record: 5
Spectral Category: technical

X-ray photoelectron spectroscopy (XPS) was used to analyze $\mathrm{Ti} / \mathrm{TiN}(001)$ bilayers grown by ultrahigh vacuum reactive magnetron sputter deposition on $\mathrm{MgO}(001)$, with an electrically floating substrate potential of $7 \mathrm{~V}$, in mixed $1: 1 \mathrm{Ar} / \mathrm{N}_{2}$ discharges maintained at a total pressure of $0.5 \mathrm{~Pa}$ $\left(3.75 \times 10^{-3}\right.$ Torr). The TiN(001) films were grown at $600{ }^{\circ} \mathrm{C}$ and the 4-ML-thick Ti overlayers at room temperature. XPS spectra were obtained using incident monochromatic $\mathrm{Al} K_{\alpha} \mathrm{X}$-radiation at $0.83401 \mathrm{~nm}$. Ti/TiN(001) Ti $2 \mathrm{p}$ spectra reveal reduced unscreened final-state satellite peaks compared to Ti 2 p spectra obtained from uncapped TiN(001) due to increased electronic screening. (C) 2012 American Vacuum Society. [http://dx.doi.org/10.1116/11.20121007]

Keywords: titanium nitride; titanium; magnetron sputtering; hard coating; transition metal nitride

PACS: 79.60.Dp, 81.15.Cd, 68.55.at, 68.55.-a

\section{INTRODUCTION}

The bulk properties of nanostructured materials (nanocomposites and nanolaminates) are often defined by the physical and electronic structure of internal interfaces which are not well understood. The $\mathrm{Si}_{3} \mathrm{~N}_{4} / \mathrm{TiN}$ nanocomposite (TiN nanocrystals encapsulated in a fully percolated $\mathrm{SiN}_{\mathrm{x}}$ tissue phase) are an example of a class of superhard materials that has attracted much attention as a model system. In work previously reported (Ref. 1), a series of 4-ML-thick overlayers were deposited by ultrahigh vacuum reactive and non-reactive magnetron sputtering onto epitaxial $\mathrm{TiN}(001)$ on $\mathrm{MgO}(001)$ in order to probe the electronic structure of $\mathrm{Si}_{3} \mathrm{~N}_{4} /$ $\mathrm{TiN}(001), \mathrm{Si} / \mathrm{TiN}(001)$, and $\mathrm{Ti} / \mathrm{TiN}(001)$ bilayer interfaces using $\mathrm{x}$-ray photoelectron spectroscopy. The 4-ML overlayer thickness is similar to reported values for the tissue layer of the $\mathrm{Si}_{3} \mathrm{~N}_{4} / \mathrm{TiN}$ nanocomposite system and is electron transparent, allowing to probe the electronic structure of the interface. It was found that these overlayer/TiN(001) interfaces have distinctly different bonding characteristics, significantly influencing the structure of the $\mathrm{Ti}$ $2 \mathrm{p}$ photoelectron spectra.

This contribution presents data from Ti, 4-ML thick, deposited onto epitaxial $\mathrm{TiN}(001)$ by ultrahigh vacuum reactive magnetron sputtering on $\mathrm{MgO}(001)$.

\footnotetext{
a) Author to whom correspondence should be addressed.
}

\section{SPECIMEN DESCRIPTION (ACCESSION \#01246)}

Host Material: $\mathrm{Ti} / \mathrm{TiN}(001)$

CAS Registry \#: 7440-32-6, 25583-20-4

Host Material Characteristics: homogeneous; solid; polycrystalline; conductor; composite; thin film

Chemical Name: titanium, titanium nitride

Source: grown in situ on $\mathrm{TiN}(001)$

Host Composition: $\mathrm{Ti} / \mathrm{TiN}$

Form: thin film

History \& Significance: In order to understand the nature of the $\mathrm{Si}_{3} \mathrm{~N}_{4} / \mathrm{TiN}$ interface, $4-\mathrm{ML}$ of $\mathrm{Ti}$ was deposited onto stoichiometric $\mathrm{TiN}(001)$ on $\mathrm{MgO}(001)$. Polycrystalline Ti was grown at room temperature and epitaxial $\mathrm{TiN}(001)$ layers were grown at $600{ }^{\circ} \mathrm{C}$ in situ by ultrahigh vacuum (UHV) reactive magnetron sputter deposition with an electrically-floating substrate potential of $-7 \mathrm{~V}$ in a growth chamber attached to an analytical chamber containing a photoelectron spectrometer. X-ray photoelectron spectra were obtained from $\mathrm{Si}_{3} \mathrm{~N}_{4} / \mathrm{TiN}(001)$ surfaces without air exposure.

As Received Condition: direct vacuum transfer from growth chamber 
Analyzed Region: same as host material

Ex Situ Preparation/Mounting: The MgO substrate was mechanically mounted to a Mo substrate heater using tungsten wire.

In Situ Preparation: Polycrystalline Ti and epitaxial TiN/MgO(001) layers were grown in a multichamber UHV system that were isolated from the analytical chamber of the instrument during growth and separated by a distance of $40 \mathrm{~cm}$ to inhibit cross contamination. The turbomolecular-pumped growth chamber had a base pressure of $<1 \times 10^{-7} \mathrm{~Pa}\left(<1 \times 10^{-9}\right.$ Torr). $\mathrm{MgO}(001)$ substrates $\left(5 \times 5 \times 0.5 \mathrm{~mm}^{3}\right)$ were annealed at $\mathrm{T}_{\mathrm{s}}=800{ }^{\circ} \mathrm{C}$ for $1 \mathrm{~h}$, a procedure known to produce wellordered $\mathrm{MgO}(001) 1 \times 1$ surfaces as determined by lowenergy electron diffraction analyses (Ref. 2). The target, a 5-cm-diameter water-cooled Ti (99.9999\%) disc, was cleaned with an $\mathrm{Ar} / \mathrm{N}_{2}$ discharge prior to film growth. The epitaxial $\mathrm{TiN}(001)$ layers were deposited first by ultrahigh vacuum reactive magnetron sputtering onto $\mathrm{MgO}(001)$ at $600{ }^{\circ} \mathrm{C}$ in mixed $1: 1 \mathrm{Ar} / \mathrm{N}_{2}$ discharges maintained at a total pressure of $0.5 \mathrm{~Pa}$ $\left(3.75 \times 10^{-3}\right.$ Torr), a target power of $100 \mathrm{~W}$, and a substrate potential of $-7 \mathrm{~V}$ (floating). This procedure produces high quality stoichiometric single crystalline TiN (Ref. 3). The 4-ML thick Ti layers were deposited next onto the epitaxial $\mathrm{TiN}(001)$ without breaking vacuum, at room temperature and a substrate bias of $-7 \mathrm{~V}$ (floating). The pure Ti target was sputtered at $100 \mathrm{~W}(304 \mathrm{~V})$ in pure $\mathrm{N}_{2}$ maintained at $0.5 \mathrm{~Pa}$ $\left(3.75 \times 10^{-3}\right.$ Torr $)$.

Pre-Analysis Beam Exposure: less than 2 min; no x-ray effects observed

Charge Control: Low energy flood gun/magnetic immersion lens combination. Filament current $=1.8 \mathrm{~A}$, charge balance $=2.5 \mathrm{~V}$, filament bias $=1 \mathrm{~V}$.

Temp. During Analysis: $300 \mathrm{~K}$

Pressure During Analysis: $<3 \times 10^{-7} \mathrm{~Pa}$

\section{INSTRUMENT DESCRIPTION}

Manufacturer and Model: Kratos Axis Ultra

Analyzer Type: spherical sector

Detector: Channeltron

Number of Detector Elements: 8

INSTRUMENT PARAMETERS COMMON TO ALL SPECTRA -

\section{- Spectrometer}

Analyzer Mode: constant pass energy

Throughput $\left(T=E^{N}\right): \mathrm{N}=0$

Excitation Source Window: $\mathrm{Al} K_{\alpha}$ (monochromated)

Excitation Source: $\mathrm{Al} K_{\alpha}$, monochromatic

Source Energy: $1486.6 \mathrm{eV}$

Source Strength: $225 \mathrm{~W}$

Source Beam Size: $2000 \mu \mathrm{m} \times 2000 \mu \mathrm{m}$

Signal Mode: multichannel direct

\section{Geometry}

Incident Angle: $54.7^{\circ}$

Source to Analyzer Angle: $54^{\circ}$

Emission Angle: $0^{\circ}$

Specimen Azimuthal Angle: $45^{\circ}$

Acceptance Angle from Analyzer Axis: $0^{\circ}$

Analyzer Angular Acceptance Width: $40^{\circ} \times 40^{\circ}$

\section{DATA ANALYSIS METHOD}

Energy Scale Correction: The binding energy scale was referenced to the Fermi Level, $E_{f},=0$. The position of $E_{f}$ was estimated by subtracting $1 / 2$ of the full width at half maximum (FWHM) from the position of the maximum of the density-ofstates, DOS, between $0-3 \mathrm{eV}$ as determined by peak fitting (DS(0.2,320)SGL(20)).

Recommended Energy Scale Shift: Accession \#s 1246-02 to 05, $2.269 \mathrm{eV}$

Peak Shape and Background Method: A Shirley background was used (Ref. 4). Asymmetric modified Gaussian/Lorentzian (product formula) line-shapes (Refs. 5 and 6) and Gaussian/ Lorentzian (sum formula) line-shapes (Ref. 6) were used to fit the Ti $2 \mathrm{p}$ and $\mathrm{N} 1 \mathrm{~s}$ spectra.

Quantitation Method: Quantification was done using peak areas from CasaXPS (version 2.3.15) region definitions for the entire peak envelope (main peaks and satellites). For simplicity, the peak amplitudes and concentrations are listed with the lowest binding energy line of each of the different elements. Sensitivity factors supplied by Kratos Analytical.

\section{ACKNOWLEDGMENTS}

This work was was carried out in the Frederick Seitz Materials Research Laboratory Central Facilities, University of Illinois.

\section{REFERENCES}

1. J. Patscheider, N. Hellgren, R. T. Haasch, I. Petrov, and J. E. Greene, Phys. Rev. B 83, 125124 (2011).

2. R. C. Powell, N.-E. Lee, Y.-W. Kim, and J. E. Greene, J. Appl. Phys. 73, 189 (1983).

3. C.-S. Shin, S. Rudenja, D. Gall, N. Hellgren, T.-Y. Lee, I. Petrov, and J. E. Greene, J. Appl. Phys. 95, 356 (2004).

4. D. A. Shirley, Phys. Rev. 55, 4709 (1972).

5. G. Wertheim, J. Electron Spectrosc. 6, 239 (1975).

6. N. Fairley and A. Carrick, The Casa Cookbook Part 1: Recipes for XPS data processing (Acolyte Science, Cheshire, UK, 2005), pp. 147-67.

7. S. Logothetidis, E. I. Meletis, and G. Kouroukis, J. Mater. Res. 14/2, 436 (1999). 


\begin{tabular}{lcccrccc}
\hline \multicolumn{7}{c}{ SPECTRAL FEATURES TABLE } \\
$\begin{array}{l}\text { Spectrum } \\
\text { ID \# }\end{array}$ & $\begin{array}{c}\text { Element/ } \\
\text { Transition }\end{array}$ & $\begin{array}{c}\text { Peak Energy } \\
\text { (eV) }\end{array}$ & $\begin{array}{c}\text { Peak Width } \\
\text { FWHM (eV) }\end{array}$ & $\begin{array}{c}\text { Peak Area } \\
\text { (eV-cts/s) }\end{array}$ & $\begin{array}{c}\text { Sensitivity } \\
\text { Factor }\end{array}$ & $\begin{array}{c}\text { Concen-tration } \\
\text { (at. \%) }\end{array}$ & $\begin{array}{c}\text { Peak } \\
\text { Assignment }\end{array}$ \\
\hline $01246-02$ & $\mathrm{O} 1 \mathrm{~s}$ & 530.8 & 1.78 & 3367 & 0.78 & 3.52 & $\ldots$ \\
$01246-03^{\mathrm{a}}$ & $\mathrm{Ti} 2 \mathrm{p}_{3 / 2}$ & 454.2 & 0.72 & 140337 & 2.001 & 57.12 & $\mathrm{Ti} / \mathrm{TiN}$ \\
$01246-03^{\mathrm{b}}$ & $\mathrm{Ti} 2 \mathrm{p}_{3 / 2}$ & 458.2 & 2.7 & $\ldots$ & $\ldots$ & $\ldots$ & $\mathrm{Ti} / \mathrm{TiN}$ \\
$01246-03^{\mathrm{a}}$ & $\mathrm{Ti} 2 \mathrm{p}_{1 / 2}$ & 460 & 0.94 & $\ldots$ & $\ldots$ & $\ldots$ & $\mathrm{Ti} / \mathrm{TiN}$ \\
$01246-03^{\mathrm{b}}$ & $\mathrm{Ti} 2 \mathrm{p}_{1 / 2}$ & 464.5 & 1.6 & $\ldots$ & $\ldots$ & $\ldots$ & $\mathrm{Ti} / \mathrm{TiN}$ \\
$01246-04$ & $\mathrm{~N} 1 \mathrm{~s}$ & 397.1 & 0.69 & 23054.3 & 0.477 & 39.36 & $\mathrm{TiN}$ \\
$01246-04^{\mathrm{c}}$ & $\mathrm{N} 1 \mathrm{~s}$ & 400.1 & 1.9 & $\ldots$ & $\ldots$ & $\ldots$ & $\mathrm{TiN}$ \\
\hline
\end{tabular}

a screened final-state

${ }^{b}$ unscreened final-state, plasmon (Ref. 7)

${ }^{c}$ plasmon (Ref. 7)

Comment to Spectral Features Table: Quantification was done using peak areas from CasaXPS (version 2.3.15) region definitions for the entire peak envelope (main peaks and satellites). For simplicity, the peak amplitudes and concentrations are listed with the lowest binding energy line of each of the different elements. Sensitivity factors supplied by Kratos Analytical.

\section{GUIDE TO FIGURES}

\begin{tabular}{|c|c|c|c|c|c|}
\hline Spectrum (Accession) \# & Spectral Region & Voltage Shift ${ }^{\star}$ & Multiplier & Baseline & Comment \# \\
\hline $1246-01$ & survey & 0 & 1 & 0 & \\
\hline $1246-02$ & O 1s & -2.269 & 1 & 0 & \\
\hline $1246-03$ & Ti $2 p$ & -2.269 & 1 & 0 & \\
\hline $1246-04$ & $\mathrm{~N} 1 \mathrm{~s}$ & -2.269 & 1 & 0 & \\
\hline $1246-05$ & valence band & -2.269 & 1 & 0 & \\
\hline
\end{tabular}

"Voltage shift of the archived (as-measured) spectrum relative to the printed figure. The figure reflects the recommended energy scale correction due to a calibration correction, sample charging, flood gun, or other phenomenon.

${ }^{* *}[\mathrm{NP}]$ signifies not published; digital spectra are archived in SSS database but not reproduced in the printed journal. 


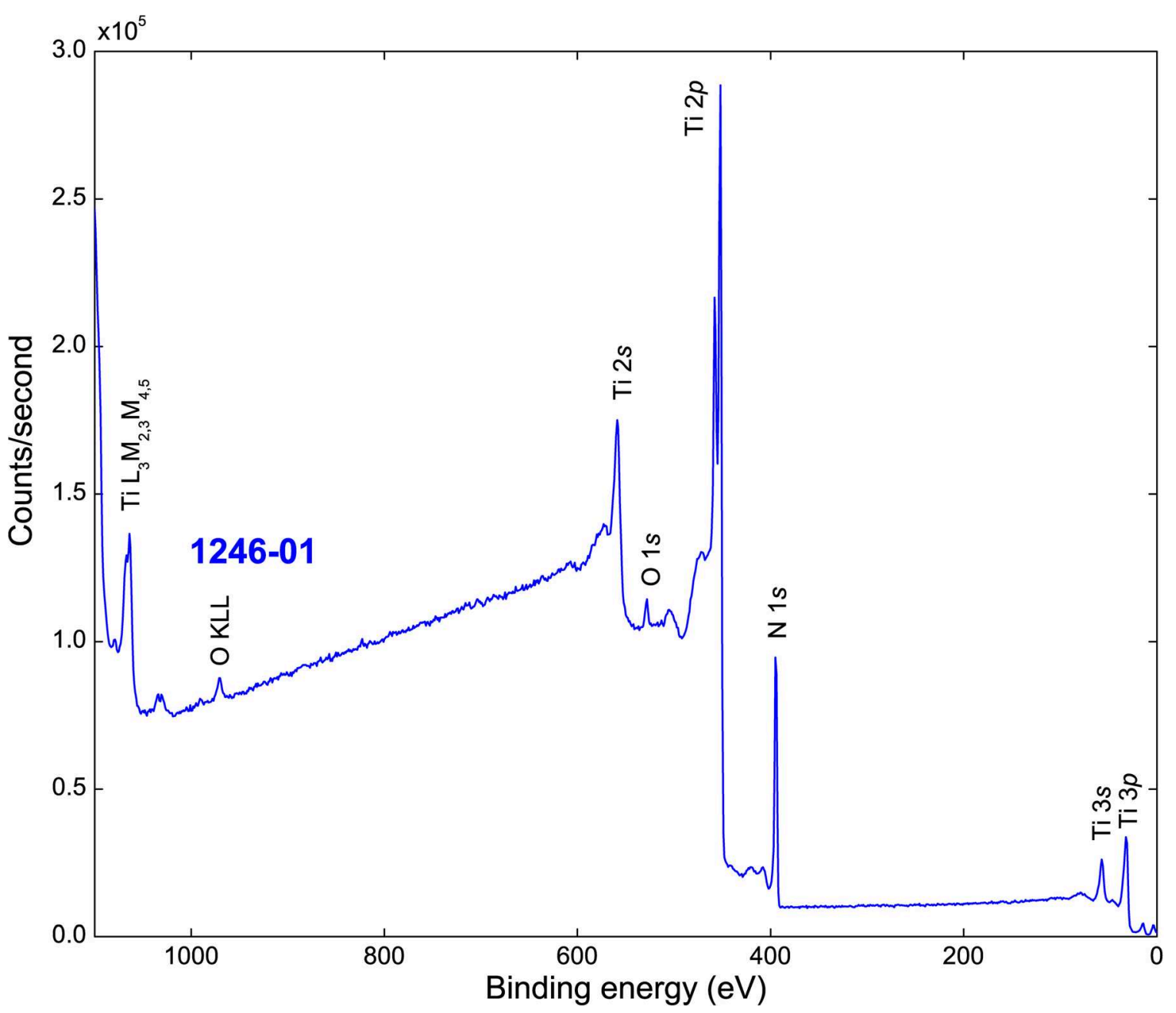

\begin{tabular}{rc}
\hline Accession \# & 01246-01 \\
\hline Host Material & Ti/TiN(001) bilayer \\
Technique & XPS \\
Spectral Region & survey \\
Instrument & Kratos Axis Ultra \\
Excitation Source & Al $K_{\alpha}$ monochromatic \\
Source Energy & $1486.6 \mathrm{eV}$ \\
Source Strength & $225 \mathrm{~W}$ \\
Source Size & $2 \mathrm{~mm} \times 2 \mathrm{~mm}$ \\
Analyzer Type & $\mathrm{spherical} \mathrm{sector}$ \\
Incident Angle & $54.7^{\circ}$ \\
Emission Angle & $0^{\circ}$ \\
Analyzer Pass Energy: & $160 \mathrm{eV}$ \\
Analyzer Resolution & $2.4 \mathrm{eV}$ \\
Total Elapsed Time & $220 \mathrm{~s}$ \\
Number of Scans & $440 \mathrm{~s}$ \\
Total Signal Accumulation Time & 2 \\
Effective Detector Width & $33.6 \mathrm{eV}$ \\
Comment & emission angle $=0^{\circ}$ \\
\hline
\end{tabular}




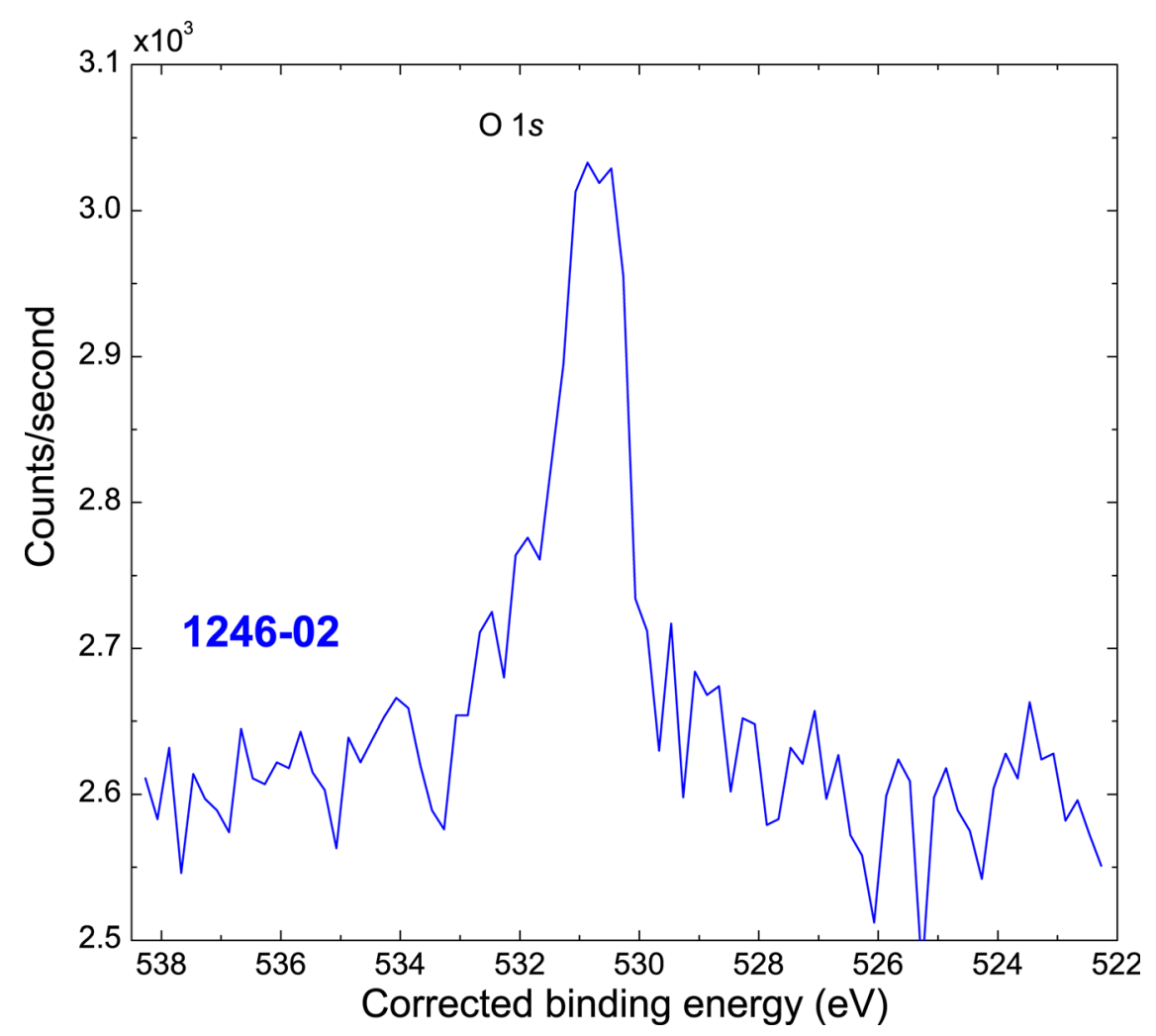

Accession \#: 01246-02

a Host Material: $\mathrm{Ti} / \mathrm{TiN}(001)$

bilayer

- Technique: XPS

- Spectral Region: $01 s$

Instrument: Kratos Axis Ultra

Excitation Source: Al $K_{\alpha}$ monochromatic

Source Energy: $1486.6 \mathrm{eV}$

Source Strength: $225 \mathrm{~W}$

Source Size: $2 \mathrm{~mm} \times 2 \mathrm{~mm}$

Analyzer Type: spherical sector Incident Angle: $54.7^{\circ}$

Emission Angle: $0^{\circ}$

Analyzer Pass Energy: $20 \mathrm{eV}$ Analyzer Resolution: $0.3 \mathrm{eV}$ Total Signal Accumulation Time: $81 \mathrm{~s}$ Total Elapsed Time: $223 \mathrm{~s}$ Number of Scans: 10 Effective Detector Width: $4.2 \mathrm{eV}$ Comment: emission angle $=0^{\circ}$

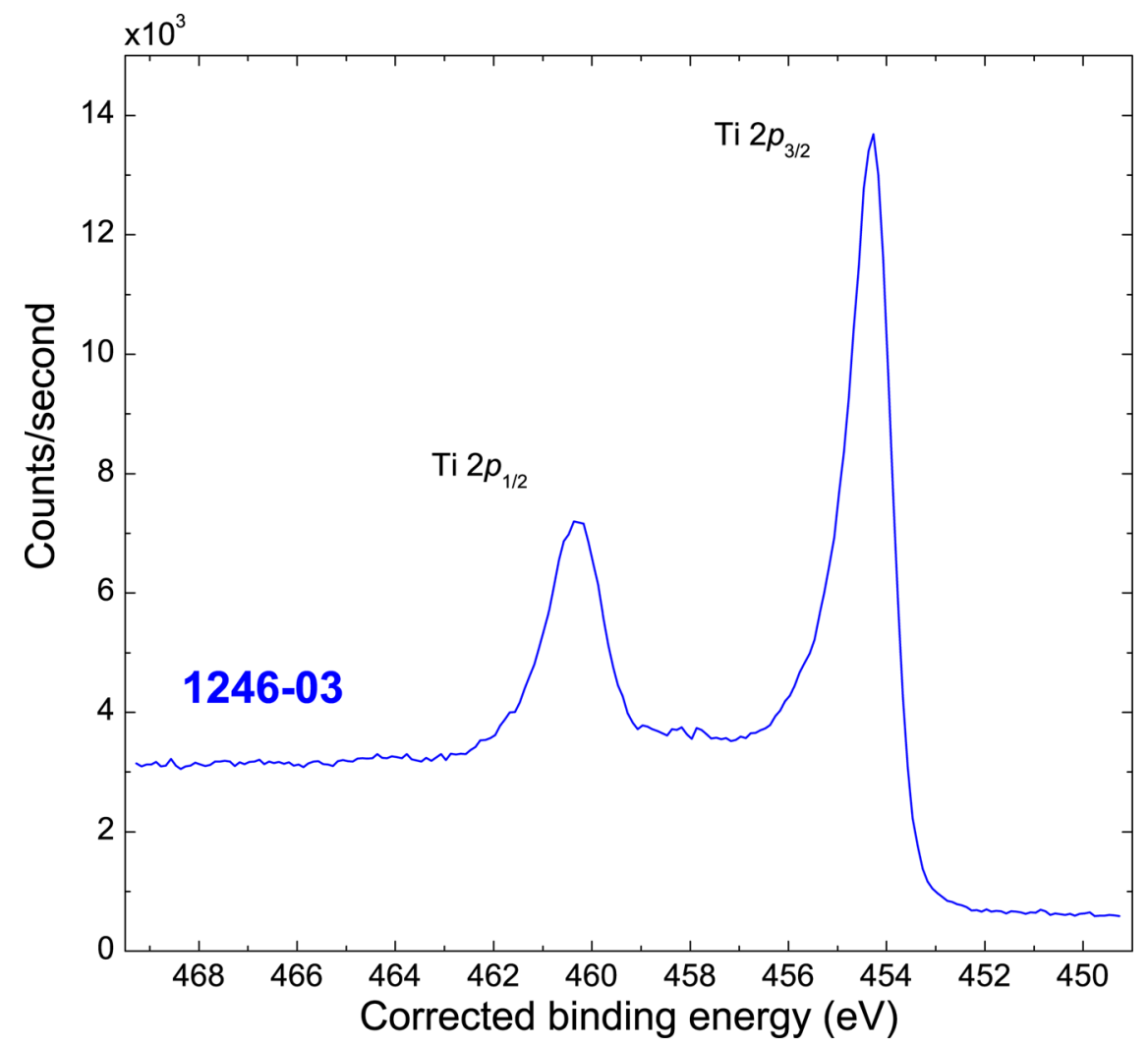

Accession \#: 01246-03

- Host Material: $\mathrm{Ti} / \mathrm{TiN}(001)$ bilayer

- Technique: XPS

- Spectral Region: Ti $2 p$

Instrument: Kratos Axis Ultra

Excitation Source: Al $K_{\alpha}$ monochromatic

Source Energy: $1486.6 \mathrm{eV}$

Source Strength: $225 \mathrm{~W}$

Source Size: $2 \mathrm{~mm} \times 2 \mathrm{~mm}$ Analyzer Type: spherical sector Incident Angle: $54.7^{\circ}$

Emission Angle: $0^{\circ}$

Analyzer Pass Energy: $20 \mathrm{eV}$ Analyzer Resolution: $0.3 \mathrm{eV}$ Total Signal Accumulation Time: $201 \mathrm{~s}$ Total Elapsed Time: $553 \mathrm{~s}$ Number of Scans: 10 Effective Detector Width: $4.2 \mathrm{eV}$ Comment: emission angle $=0^{\circ}$ 

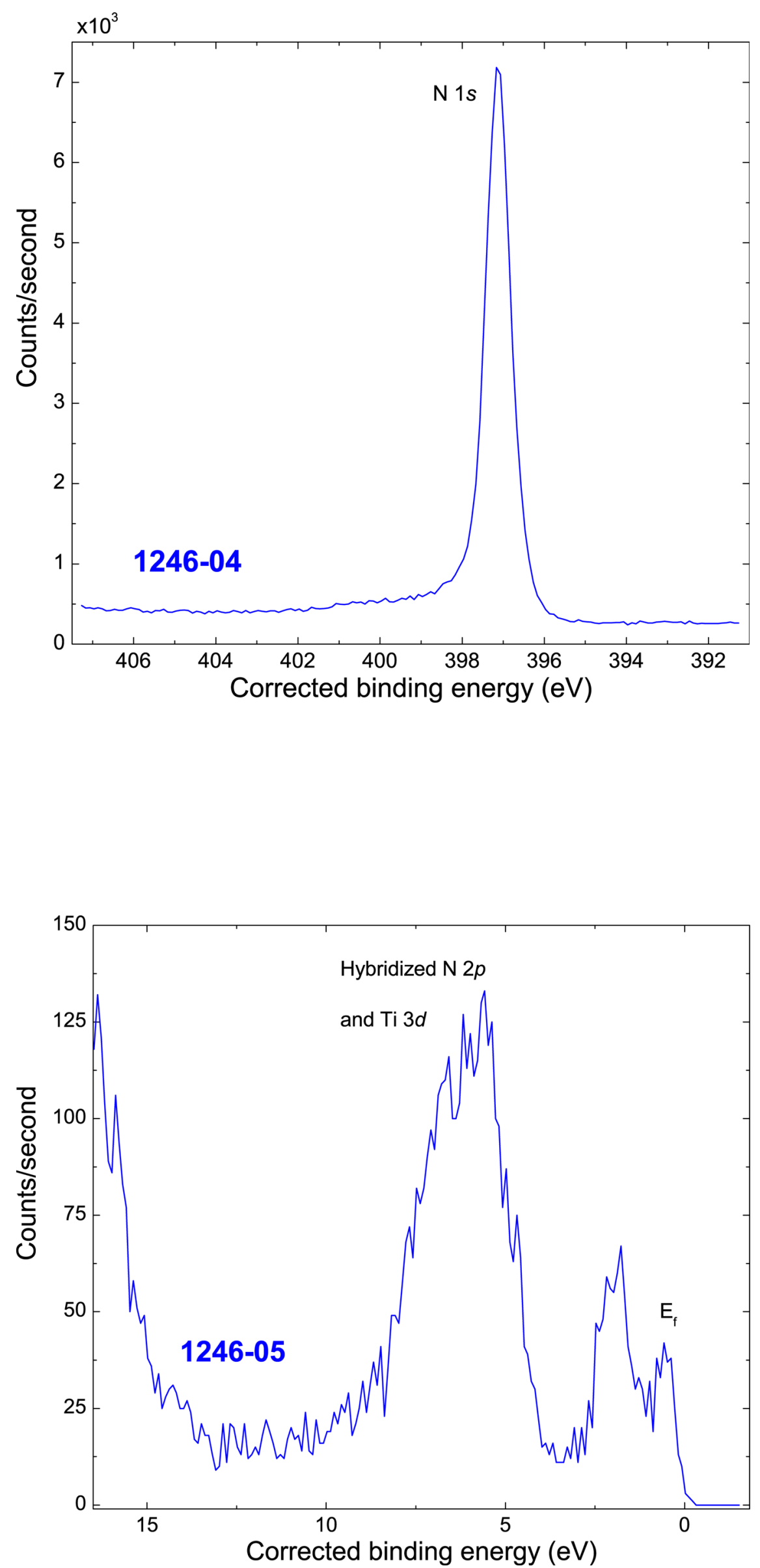

Accession \#: 01246-04

a Host Material: Ti/TiN(001) bilayer

- Technique: XPS

- Spectral Region: N $1 s$

Instrument: Kratos Axis Ultra

Excitation Source: Al $K$ monochromatic

Source Energy: $1486.6 \mathrm{eV}$

Source Strength: $225 \mathrm{~W}$

Source Size: $2 \mathrm{~mm} \times 2 \mathrm{~mm}$

Analyzer Type: spherical sector Incident Angle: $54.7^{\circ}$

Emission Angle: $0^{\circ}$

Analyzer Pass Energy: $20 \mathrm{eV}$ Analyzer Resolution: $0.3 \mathrm{eV}$ Total Signal Accumulation Time: $161 \mathrm{~s}$ Total Elapsed Time: $443 \mathrm{~s}$ Number of Scans: 10 Effective Detector Width: $4.2 \mathrm{eV}$ Comment: emission angle $=0^{\circ}$
Accession \#: 01246-05

- Host Material: Ti/TiN(001) bilayer

- Technique: XPS

- Spectral Region: valence band Instrument: Kratos Axis Ultra Excitation Source: Al $K_{\alpha}$ monochromatic

Source Energy: $1486.6 \mathrm{eV}$

Source Strength: $225 \mathrm{~W}$

Source Size: $2 \mathrm{~mm} \times 2 \mathrm{~mm}$ Analyzer Type: spherical sector Incident Angle: $54.7^{\circ}$

Emission Angle: $0^{\circ}$

Analyzer Pass Energy: $20 \mathrm{eV}$ Analyzer Resolution: $0.3 \mathrm{eV}$ Total Signal Accumulation Time: $181 \mathrm{~s}$ Total Elapsed Time: $498 \mathrm{~s}$ Number of Scans: 10 Effective Detector Width: $4.2 \mathrm{eV}$ Comment: emission angle $=0^{\circ}$ 